\title{
Reversal of Cocaine-Associated Synaptic Plasticity in Medial Prefrontal Cortex Parallels Elimination of Memory Retrieval
}

\author{
James M Otis ${ }^{1,2}$ and Devin Mueller ${ }^{*, 1,3}$ \\ 'Department of Psychology, University of Wisconsin-Milwaukee, Milwaukee, WI, USA; ${ }^{2}$ Department of Psychiatry, University of North Carolina- \\ Chapel Hill, Chapel Hill, NC, USA; ${ }^{3}$ Department of Basic Sciences, Neuroscience Division, Ponce Health Sciences University-School of Medicine, \\ Ponce Research Institute, Ponce, Puerto Rico
}

\begin{abstract}
Addiction is characterized by abnormalities in prefrontal cortex that are thought to allow drug-associated cues to drive compulsive drug seeking and taking. Identification and reversal of these pathologic neuroadaptations are therefore critical for treatment of addiction. Previous studies using rodents reveal that drugs of abuse cause dendritic spine plasticity in prelimbic medial prefrontal cortex (PL-mPFC) pyramidal neurons, a phenomenon that correlates with the strength of drug-associated memories in vivo. Thus, we hypothesized that cocaine-evoked plasticity in PL-mPFC may underlie cocaine-associated memory retrieval, and therefore disruption of this plasticity would prevent retrieval. Indeed, using patch clamp electrophysiology we find that cocaine place conditioning increases excitatory presynaptic and postsynaptic transmission in rat PL-mPFC pyramidal neurons. This was accounted for by increases in excitatory presynaptic release, pairedpulse facilitation, and increased AMPA receptor transmission. Noradrenergic signaling is known to maintain glutamatergic plasticity upon reactivation of modified circuits, and we therefore next determined whether inhibition of noradrenergic signaling during memory reactivation would reverse the cocaine-evoked plasticity and/or disrupt the cocaine-associated memory. We find that administration of the $\beta$-adrenergic receptor antagonist propranolol before memory retrieval, but not after (during memory reconsolidation), reverses the cocaine-evoked presynaptic and postsynaptic modifications in PL-mPFC and causes long-lasting memory impairments. Taken together, these data reveal that cocaine-evoked synaptic plasticity in PL-mPFC is reversible in vivo, and suggest a novel strategy that would allow normalization of prefrontal circuitry in addiction.

Neuropsychopharmacology (2017) 42, 2000-2010; doi: I 0.1038/npp.2017.90; published online 24 May 20I7
\end{abstract}

\section{INTRODUCTION}

Presentation of contextual cues associated with previous drug use drives compulsive drug seeking and taking (Childress et al, 1986; Heather et al, 1991), creating a significant obstacle in recovery from addiction. Disruption of the associative memories that allow cues to trigger drug seeking would alleviate this compulsion, although the neuroadaptations that underlie and maintain drugassociated memories remain unclear. Previous studies show that abnormalities in prefrontal cortex (PFC) are associated with addiction. For example, drug-associated cues are particularly capable of activating the PFC in addicts, and the level of PFC activation correlates with reported drug cravings (Grant et al, 1996; Grüsser et al, 2004; Kilts et al, 2001). Furthermore, in rodent studies, drug exposure causes dendritic spine gain in pyramidal neurons of the prelimbic medial PFC (PL-mPFC; Robinson et al, 2001; Robinson and

*Correspondence: Dr D Mueller, Department of Basic Sciences, Neuroscience Division, Ponce Health Sciences University-School of Medicine, Ponce Research Institute, P.O. Box 7004, Ponce 00732-7004, Puerto Rico, Tel: + I 7878402575 Ext. 2588, Fax: + I 787844 1980, E-mail: dmueller@psm.edu

Received 21 December 2016; revised 26 April 2017; accepted 27 April 2017; accepted article preview online 3 May 2017
Kolb, 2004,1999), an effect that parallels the formation of drug-associated memories in vivo (Muñoz-Cuevas et al, 2013). Thus, addiction is associated with adaptations in PFC, which may underlie drug-associated memories, but whether these adaptations could be reversed in vivo is unknown.

Previously, we found that inhibition of $\beta$-adrenergic receptor ( $\beta$-AR) signaling can persistently impair the expression of drug-associated memories (Otis and Mueller, 2011; Otis et al, 2013,2014a). Specifically, we observed that systemic administration of $\beta$-AR antagonists before a single cocaine-conditioned place preference (CPP) memory retrieval test prevents CPP expression during that test and during subsequent treatment-free tests. This impairment is long lasting, and provides protection against cocaine-induced reinstatement. In addition, CPP behavioral deficits are not due to $\beta$-AR antagonism in the peripheral nervous system (Otis and Mueller, 2011), but can be replicated through local infusions of $\beta$-AR antagonists into the PL-mPFC (Otis et al, 2013). Importantly, systemic or intra-PL-mPFC injections of $\beta$-AR antagonists do not influence locomotion, do not induce an affective state capable of inducing a CPP or aversion, and have no effect on memory when administered after, rather than before, a single CPP retrieval test (Otis and Mueller, 2011; Otis et al, 2013). Taken together, $\beta$-AR blockade during memory retrieval eliminates the expression of a cocaine- 
associated memory. However, whether $\beta$-AR blockade also eliminates neuroadaptations associated with cocaine exposure and learning is unknown.

Here we combine in vivo neuropharmacology with ex vivo patch-clamp electrophysiology, and find that cocaine place conditioning increases both presynaptic release and postsynaptic efficacy at glutamatergic synapses of PL-mPFC pyramidal neurons. Furthermore, we find that inhibition of $\beta$-AR signaling before memory retrieval, but not after (during memory reconsolidation), reverses the cocaineevoked neuroadaptations in PL-MPFC and causes longlasting disruption of the cocaine-associated memory. Taken together, these data reveal that cocaine-evoked synaptic plasticity in PL-mPFC is reversible in vivo, and suggest a novel strategy that would allow normalization of prefrontal circuitry in addiction.

\section{MATERIALS AND METHODS}

\section{Subjects}

All behavioral protocols were approved by the Institutional Animal Care and Use Committee at the University of Wisconsin-Milwaukee. Male Long-Evans rats (3-5 months) were housed individually in clear plastic cages with water and rat chow (Harlan Laboratories) available ad libitum. Rats were maintained on a 14-h light/dark schedule (lights on at 0700 hours), and all behavioral protocols were completed during the light cycle.

\section{Drugs}

Cocaine $\mathrm{HCl}$ (National Institute on Drug Abuse) was dissolved in sterile saline $(0.9 \% \mathrm{NaCl})$ and administered at a dose of $10 \mathrm{mg} / \mathrm{kg}$ (i.p.). The $\beta$-AR antagonist propranolol (Sigma-Aldrich) was dissolved in sterile saline $(0.9 \%)$ and administered at a dose of $10 \mathrm{mg} / \mathrm{kg}$ (i.p.).

\section{Conditioning and Testing}

Place conditioning and testing were performed as described previously in detail (Otis and Mueller, 2011). Briefly, the conditioning apparatus was composed of two distinguishable conditioning chambers separated by a smaller center chamber. Rats were given access to all three chambers during a 15-min baseline test to assess initial chamber biases. Overall, rats spent equivalent time in each conditioning chamber, but less time in the smaller center chamber. Thus, an unbiased conditioning procedure was used, wherein rats were assigned to receive cocaine in one chamber and saline in the other in a pseudorandom and counterbalanced fashion. Conditioning took place during eight daily sessions, wherein rats received cocaine $(10 \mathrm{mg} / \mathrm{kg}$, i.p.) or saline (i.p., four injections of each) in an alternating manner immediately before confinement within their corresponding conditioning chamber for $20 \mathrm{~min}$. Rats were next given a 2-day break before daily CPP retrieval tests. During the CPP tests, rats were allowed full access to all three chambers for $15 \mathrm{~min}$. Systemic injections aimed to induce erasure of the cocaineassociated memory were administered before (to target retrieval) or after (to target reconsolidation) the first CPP test, but not the second CPP test. Overall, we found no effect of pretest propranolol injections on time spent in the center chamber during the first and second CPP test (saline-treated rats, $n=8$; propranolol-treated rats, $n=7$; ANOVA, effect of group: $\left.\mathrm{F}_{1,13}=0.25, p=0.63\right)$. Thus, to simplify data analysis and illustration, place conditioning data were analyzed using CPP scores, as quantified by time spent in the previously cocaine paired chamber minus time in the saline-paired chamber, subtracted from preconditioning test scores. Differences in CPP scores were analyzed using two-way ANOVAs (group $\times$ test), and planned comparison post hoc analyses were performed to compare differences in CPP scores between the two groups for each test.

\section{Electrophysiology}

Rats were anesthetized with pentobarbital (50 mg/kg, i.p.) and brains were rapidly extracted and sectioned $(350 \mu \mathrm{m})$ in ice-cold $\left(0-2{ }^{\circ} \mathrm{C}\right)$ oxygenated $\left(20: 1\right.$ ratio of $\left.\mathrm{O}_{2}: \mathrm{CO}_{2}\right)$ artificial cerebral spinal fluid (aCSF; in mM: $124 \mathrm{NaCl}, 2.8$ $\mathrm{KCl}, 1.25 \mathrm{NaH}_{2} \mathrm{PO}_{4}, 2 \mathrm{MgSO}_{4}, 2 \mathrm{CaCl}_{2}, 26 \mathrm{NaHCO}_{3}$, and 20 glucose) using a vibrating blade (Leica, VT1200). Slices recovered in warm aCSF $\left(32^{\circ} \mathrm{C}\right)$ for a minimum of $30 \mathrm{~min}$ before recordings. During recordings, slices were continuously perfused with aCSF $(2 \mathrm{ml} / \mathrm{min})$, in the presence of picrotoxin $(100 \mu \mathrm{M})$ for blockade of GABA-mediated currents. PL-mPFC pyramidal neurons were visualized using differential interference contrast through a liquid-immersion lens mounted on an upright light microscope (Nikon Instruments). Layer V/VI PL-mPFC pyramidal neurons were identified based on morphology and electrophysiological characteristics, as described previously (Otis et al, $2013,2014 b$ ). Whole-cell recordings were obtained using low-resistance borosilicate pipettes (2-5 M $\Omega$ ) containing a potassium gluconate-based internal solution (in mM: $110 \mathrm{~K}$ gluconate, $20 \mathrm{KCl}, 10 \mathrm{HEPES}, 2 \mathrm{MgCl}_{2}$, $2 \mathrm{ATP}, 0.3 \mathrm{GTP}, 10$ phosphocreatine, $0.2 \%$ biocytin; $\mathrm{pH} 7.3$, and mOsm 280). Current-clamp and voltage-clamp recordings were made using a signal amplifier (MultiClamp 700B, Molecular Devices) and digitizer (Digidata 1440A, Molecular Devices). Throughout all recordings, the liquid-liquid junction potential of $13 \mathrm{mV}$ was compensated through voltage subtraction.

Current-clamp recordings were used for characterization of intrinsic neuronal excitability. Rheobase was first evaluated by applying a series of $1 \mathrm{~s}$ hyperpolarizing and depolarizing current steps $(10 \mathrm{pA}$ steps starting at $-40 \mathrm{pA})$. Rheobase of each neuron was measured as the minimum stimulation intensity necessary for action potential initiation. Action potential frequency was also examined through application of large depolarizing steps $(0-500 \mathrm{pA}$, $50 \mathrm{pA}$ steps) while holding the neurons at resting membrane potential.

Voltage-clamp recordings were used for characterization of synaptic plasticity as previously described (Otis et al, $2014 \mathrm{~b})$. First, sEPSCs were recorded by holding the neurons for a minimum of $2 \mathrm{~min}$ at $-80 \mathrm{mV}$. sEPSC frequency and amplitude were then analyzed using a template analysis (Clampfit 10.3). Next, we evaluated the mechanism for changes in sEPSC amplitude and frequency through stimulation-evoked recordings. Synaptic currents were evoked through presynaptic stimulation using a bipolar concentric electrode (FHC) placed $250 \mu \mathrm{m}$ proximal to the cell soma. Before starting the evoked experiments, a series of 
a

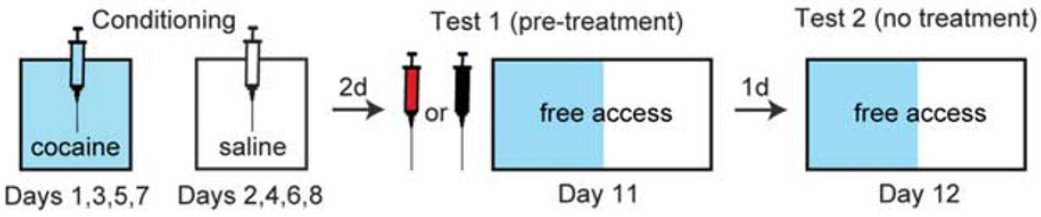

b

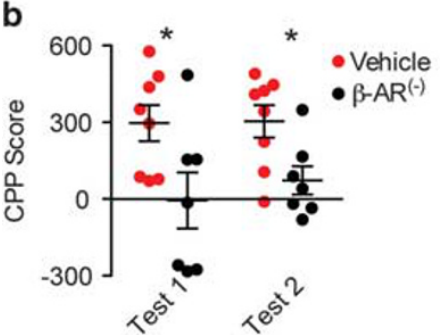

C

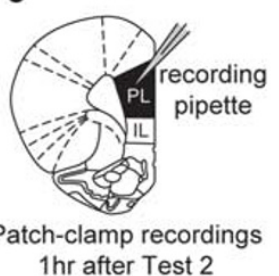

d

PL-mPFC pyramidal neuron

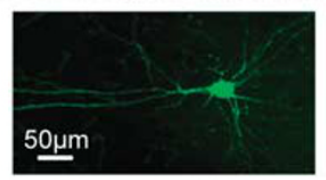

$1 \mathrm{hr}$ after Test 2

e

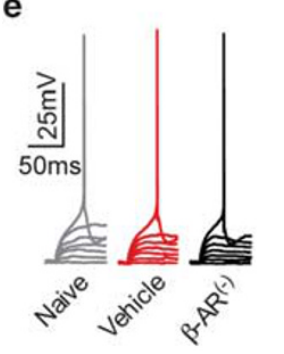

i

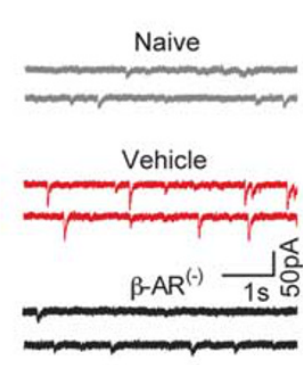

f
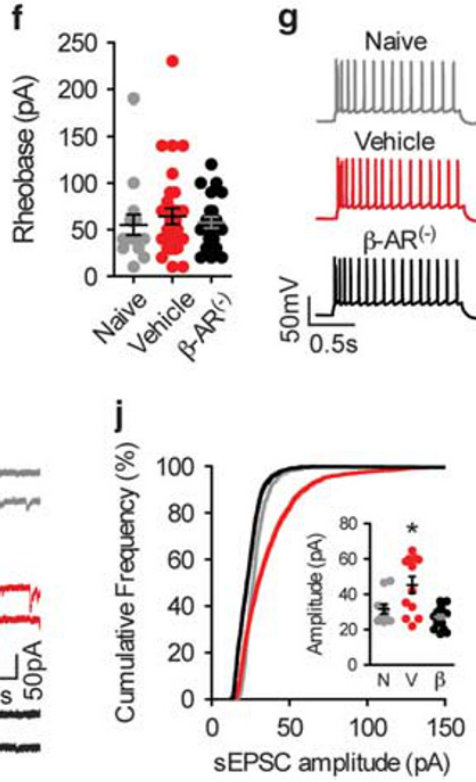

g

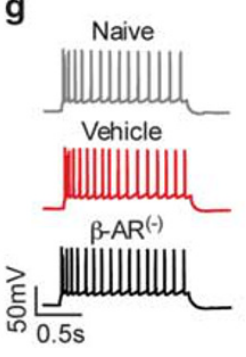

h
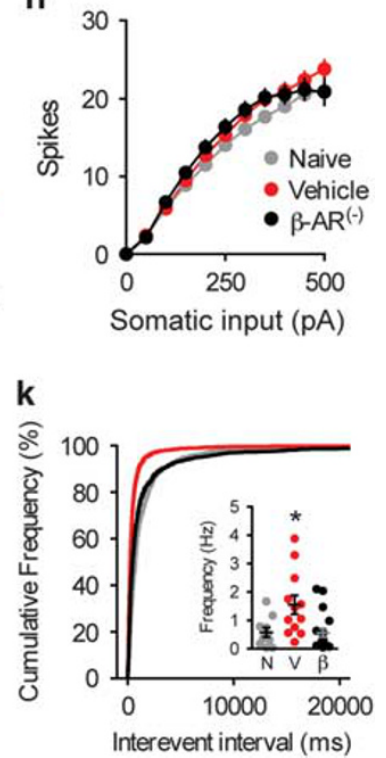

Figure I Cocaine conditioning increases spontaneous excitatory synaptic transmission in PL-mPFC pyramidal neurons: reversal by $\beta$-AR blockade during memory retrieval. (a) Schematic illustrating behavioral design of the experiment, wherein saline (red) or propranolol (black) was injected before the first CPP test. (b) Dot plots revealing that propranolol injections, but not vehicle injections, before the first CPP test prevented CPP expression during that test (test I) and during a subsequent propranolol-free test (test 2). (c) Coronal section illustrating patch-clamp recordings in PL-mPFC. (d) Representative example of a biocytin-filled PL-mPFC pyramidal neuron. (e) Representative waveforms for rheobase recordings. (f) Dot plots revealing that no differences in rheobase were found between groups. (g) Representative waveforms for excitability sweep recordings. (h) Line graphs revealing that no differences in excitability sweep recordings were found between groups. (i) Representative waveforms for sEPSC recordings. (j) Cumulative frequency distribution and dot plots (inset) revealing that neurons from vehicle-treated rats had significantly higher sEPSC amplitudes as compared with neurons from naive and propranolol-treated rats. (k) Cumulative frequency distribution and dot plots (inset) revealing that neurons from vehicle-treated rats had significantly higher sEPSC frequencies as compared with neurons from naive and propranolol-treated rats. $* p<0.05$ vs control. Lines in dot plots represent the mean \pm SE.

ascending stimulation intensities $(2.5-50 \mu \mathrm{A} ; 0.67 \mathrm{~Hz})$ were applied while holding the neuron at $-80 \mathrm{mV}$ until the maximum monosynaptic EPSC was evoked. Next, we recorded AMPAr-mediated EPSCs by evoking 20 EPSCs at $-80 \mathrm{mV}$, a voltage that prevents NMDAr currents due to magnesium blockade. We also recorded EPSCs using the same stimulation intensity while holding the neurons at $-35 \mathrm{mV}$, a voltage that unleashes inward-rectifying NMDAr currents in mPFC pyramidal neurons (Otis et al, 2014b). Because the rectification of AMPAr currents between - 80 and $-35 \mathrm{mV}$ is linear, dividing the peak amplitude of evoked EPSCs at $-80 \mathrm{mV}$ (AMPA) by that at $-35 \mathrm{mV}$ (NMDA) is a direct index of the AMPA/NMDA ratio. We have described this particular method as the 'voltage AMPA/NMDA ratio' throughout the paper. We confirmed this method by also recording the AMPA/NMDA ratio using a pharmacological method, sometimes in the same PL-mPFC neurons. To do this, evoked glutamate-mediated EPSCs were recorded at $-35 \mathrm{mV}$ for $5 \mathrm{~min}$. Next, the NMDAr antagonist APV $(50 \mu \mathrm{M})$ was applied for $20 \mathrm{~min}$. We then subtracted residual EPSCs (AMPA) from baseline (AMPA+NMDA) to calculate NMDAr EPSCs. This 'pharmacological AMPA/NMDA ratio' was highly correlated with that of the voltage AMPA/NMDA ratio $(r=0.93, p<0.001)$, confirming that these techniques accurately depict the contribution of AMPAr- and NMDArmediated current to the evoked EPSCs. We also assessed 
presynaptic plasticity by evoking a pair of EPSCs separated by $250 \mathrm{~ms}$ while holding neurons at $-80 \mathrm{mV}$. To calculate the paired pulse ratio, the amplitude of the second EPSC was divided by the amplitude of the first EPSC. All electrophysiological data were quantified using Clampfit 10.3, and ANOVA followed by planned comparison post hoc tests were used to analyze differences between naive and conditioned groups.

\section{RESULTS}

\section{$\beta$-AR Blockade Before CPP Memory Retrieval Causes Persistent Memory Impairment}

To examine cocaine-associated memory in vivo-and to allow subsequent analysis of cocaine-evoked plasticity ex vivo -rats were trained using a cocaine-CPP procedure. Rats were conditioned to associate one chamber, but not another, with cocaine (Figure 1a, left). Two days after conditioning, rats were given full access to both conditioning chambers during the first CPP retrieval test. To determine how noradrenergic signaling regulates cocaine-related synaptic plasticity and memory, rats were treated with either vehicle or the $\beta$-AR antagonist propranolol $\left(\beta-\mathrm{AR}^{(-)}\right)$before this test (Figure 1a, middle). One day after the drug treatment test, rats were given another CPP retrieval test without drug treatment (Figure 1a, right). Consistent with previous reports, rats treated with propranolol showed significantly reduced CPP scores during both the propranolol treatment test and the subsequent propranolol-free test (Figure 1b; vehicle-treated, $n=8$ rats; propranolol-treated, $n=7$ rats). Two-way ANOVA revealed a significant effect of group $\left(\mathrm{F}_{1,13}=9.59, \quad p<0.01\right)$, but no group by test interaction $\left(\mathrm{F}_{1,13}=0.30, p=0.60\right)$, and post hoc analyses confirmed that propranolol-treated rats had significantly reduced CPP scores during both the propranolol treatment test (test 1; $p=0.03$ ) and propranolol-free test (test $2 ; p=0.02$ ). Thus, $\beta$-AR blockade induced a persistent deficit in retrieval of the cocaine-associated memory. Despite this, how cocaine conditioning and $\beta$-AR blockade during retrieval changes the functional activity of PL-mPFC pyramidal neurons is unclear.

\section{$\beta$-AR Blockade Before CPP Memory Retrieval Reverses Cocaine-Evoked Adaptations in PL-mPFC Spontaneous Synaptic Transmission}

To characterize functional intrinsic and synaptic plasticity in PL-mPFC pyramidal neurons following cocaine conditioning, rats were killed after the second CPP retrieval test for patch-clamp electrophysiological recordings (Figure 1c and d). Data revealed that cocaine conditioning did not influence the intrinsic excitability of PL-mPFC pyramidal neurons, as measured through rheobase (the minimum current required to evoke an action potential; Figure 1e and f) and current-evoked excitability sweeps (Figure $1 \mathrm{~g}$ and $\mathrm{h}$ ). For rheobase recordings (naive, $n=15$ cells, 5 rats; vehicletreated, $n=31$ cells, 7 rats; propranolol-treated, $n=24$ cells, 6 rats), one-way ANOVA revealed no effect of group $\left(\mathrm{F}_{2,67}=0.41, p=0.66\right)$. For current-evoked excitability sweeps (naive, $n=15$ cells, 5 rats; vehicle-treated, $n=31$ cells, 7 rats; propranolol-treated, $n=24$ cells, 6 rats), two-way ANOVA revealed no effect of group $\left(\mathrm{F}_{2,670}=0.93, p=0.40\right)$, or group by input interaction $\left(\mathrm{F}_{20,670}=1.49, p=0.08\right)$. Thus, cocaine conditioning did not significantly influence the intrinsic excitability of PL-mPFC pyramidal neurons.

Next, we determined whether cocaine conditioning influenced excitatory synaptic transmission in PL-mPFC pyramidal neurons. To do so, we recorded spontaneous excitatory postsynaptic currents (sEPSCs; Figure 1i), and measured the average amplitude and frequency of those currents to index postsynaptic (sEPSC amplitude) and presynaptic (sEPSC frequency) plasticity. Cocaine conditioning increased both sEPSC amplitude and frequency in PL-mPFC pyramidal neurons, an effect that was reversed by propranolol treatment before the first CPP retrieval test. For sEPSC amplitude (naive, $n=12$ cells, 3 rats; vehicletreated, $n=10$ cells, 4 rats; propranolol-treated, $n=18$ cells, 4 rats), one-way ANOVA revealed a significant effect of group $\left(\mathrm{F}_{2,37}=10.92, \quad p<0.001\right)$, and post hoc analyses revealed that neurons from vehicle-treated rats had significantly higher sEPSC amplitudes as compared with neurons from naive $(p=0.03)$ and propranolol-treated rats $(p<0.001)$. For sEPSC frequency (naive, $n=12$ cells, 3 rats; vehicle-treated, $n=10$ cells, 4 rats; propranolol-treated, $n=18$ cells, 4 rats), one-way ANOVA revealed a significant effect of group $\left(\mathrm{F}_{2,37}=6.33, p<0.01\right)$, and post hoc analyses revealed that neurons from vehicle-treated rats had significantly higher sEPSC frequencies as compared with neurons from naive rats $(p=0.02)$ and propranololtreated rats $(p<0.01)$. Collectively, these data suggest that cocaine conditioning increases postsynaptic and presynaptic drive on PL-mPFC pyramidal neurons, and this plasticity is reversed by $\beta$-AR blockade during memory retrieval.

\section{$\beta$-AR Blockade Before CPP Memory Retrieval Reverses Cocaine-Evoked Paired-Pulse Facilitation}

We found that cocaine conditioning elevated spontaneous excitatory synaptic drive onto PL-mPFC pyramidal neurons, but the mechanism for this plasticity was unclear. To address this, in the next set of experiments, we performed stimulation-evoked synaptic recordings (Figure $2 \mathrm{a}$ ) in rats that had been exposed to the same conditioning procedures as presented above (see Figure 1a). First, we evaluated neural facilitation, a form of short-term presynaptic plasticity (millisecond time scale) that elevates presynaptic transmitter release through residual presynaptic $\mathrm{Ca}^{2+}$ entry during presynaptic burst firing (for review, see Zucker and Regehr, 2002). To do this, we evoked pairs of presynaptic pulses (Figure 2b; naive, $n=9$ cells, 3 rats; vehicle-treated, $n=9$ cells, 3 rats; propranolol-treated, $n=17$ cells, 4 rats). We found that cocaine conditioning caused paired-pulse facilitation in PL-mPFC pyramidal neurons, suggesting that a burst of action potentials at PL-mPFC inputs result in greater facilitation of neurotransmitter release in conditioned animals as compared with controls. Furthermore, this presynaptic plasticity was reversed through propranolol treatment before the first CPP retrieval test (Figure 2c). One-way ANOVA revealed a significant effect of group $\left(\mathrm{F}_{2,31}=9.93, p<0.001\right)$, and post hoc analyses revealed that neurons from vehicle-treated rats had significantly higher paired-pulse ratios as compared with neurons from naive 
a

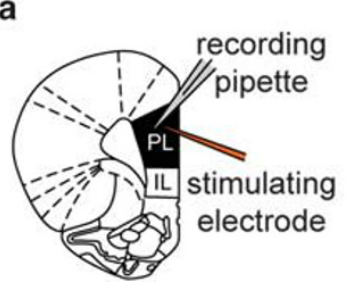

Evoked recordings $1 \mathrm{hr}$ after Test 2

d

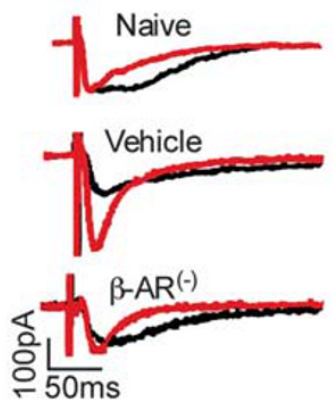

g

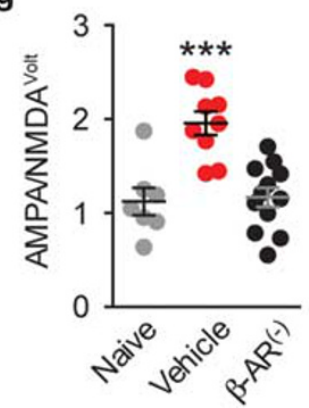

b

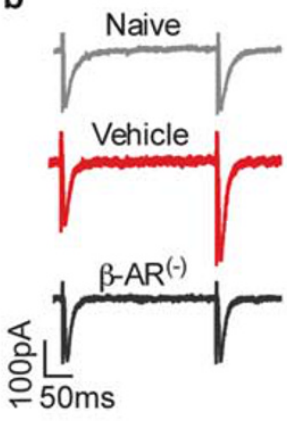

e

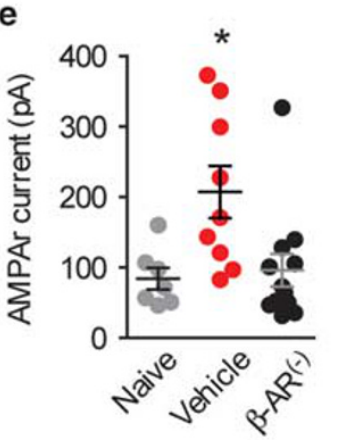

h

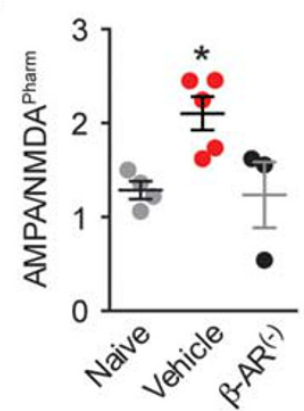

C

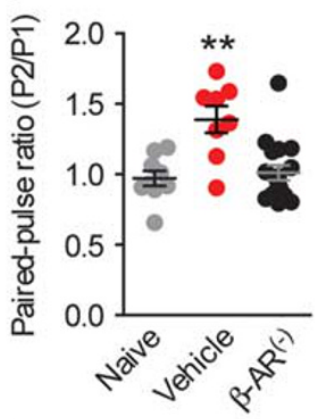

f

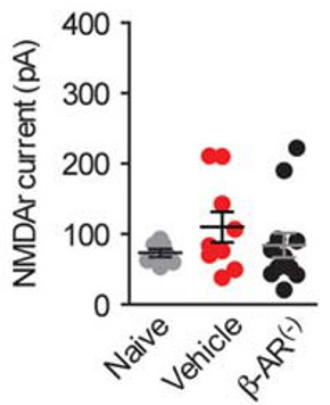

i

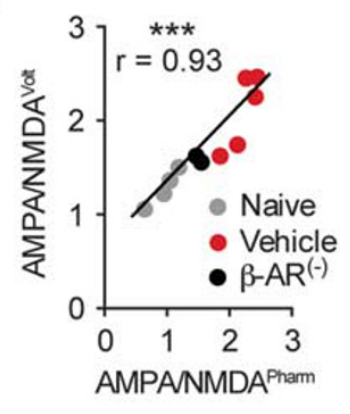

Figure 2 Cocaine conditioning increases evoked AMPA receptor transmission in PL-mPFC pyramidal neurons: reversal by $\beta$-AR blockade during memory retrieval. (a) Coronal section illustrating patch-clamp recordings of stimulation-evoked synaptic currents in PL-mPFC. (b) Representative waveforms for pairedpulse ratio recordings. (c) Dot plots revealing that neurons from vehicle-treated rats had significantly higher paired-pulse ratios as compared with neurons from naive and propranolol-treated rats. (d) Representative waveforms for AMPA and NMDA receptor-mediated currents. (e) Dot plots revealing that neurons from vehicle-treated rats had significantly higher AMPA receptor-mediated currents as compared with neurons from naive rats and propranolol-treated rats. ( $f$ ) Dot plots revealing no group differences for NMDA receptor-mediated currents. (g) Dot plots revealing that neurons from vehicle-treated rats had significantly higher AMPA/NMDA ${ }^{\text {volt }}$ as compared with neurons from naive rats and propranolol-treated rats. (h) Dot plots revealing that neurons from vehicle-treated rats had significantly higher AMPA/NMDA Pharm as compared with neurons from naive and propranolol-treated rats. (i) Correlation between AMPA/ $\mathrm{NMDA}^{\text {Volt }}$ and AMPA/NMDA ${ }^{\text {Pharm }}$ reveals a significant relationship between these two AMPA/NMDA ratios. * $p<0.05$ vs control; *** $<0.0$ I vs control; **** $p<0.00$ I vs control. Lines in dot plots represent the mean \pm SE.

$(p=0.001)$ and propranolol-treated rats $(p=0.001)$. Collectively, these data reveal that cocaine conditioning increases neural facilitation in PL-mPFC pyramidal neurons, and this plasticity is reversed by $\beta$-AR blockade during memory retrieval.

\section{$\beta$-AR Blockade Before CPP Memory Retrieval Reverses Cocaine-Evoked AMPA Receptor Plasticity}

Cocaine conditioning elevates presynaptic release and neural facilitation of PL-mPFC pyramidal neurons, but how cocaine conditioning elevates postsynaptic transmission in these neurons is unclear. To address this, we measured AMPA receptor-mediated EPSCs in PL-mPFC pyramidal neurons
(Figure 2d; naive, $n=7$ cells, 3 rats; vehicle-treated, $n=9$ cells, 4 rats; propranolol-treated, $n=12$ cells, 4 rats). Cocaine conditioning increased the amplitude of AMPA receptormediated EPSCs, an effect that was reversed by propranolol treatment before the first CPP retrieval test (Figure 2e). Oneway ANOVA revealed a significant effect of group $\left(\mathrm{F}_{2,25}=5.71, p<0.01\right)$, and post hoc analyses revealed that neurons from cocaine-treated rats had significantly higher AMPA receptor-mediated EPSCs as compared with neurons from naive $(p=0.01)$ and propranolol-treated rats $(p=0.02)$. In contrast, we found that cocaine conditioning had no effect on NMDA receptor currents (Figure 2f; naive, $n=7$ cells, 3 rats; vehicle-treated, $n=9$ cells, 4 rats; propranolol-treated, $n=12$ cells, 4 rats), as one-way ANOVA revealed no effect of 
a
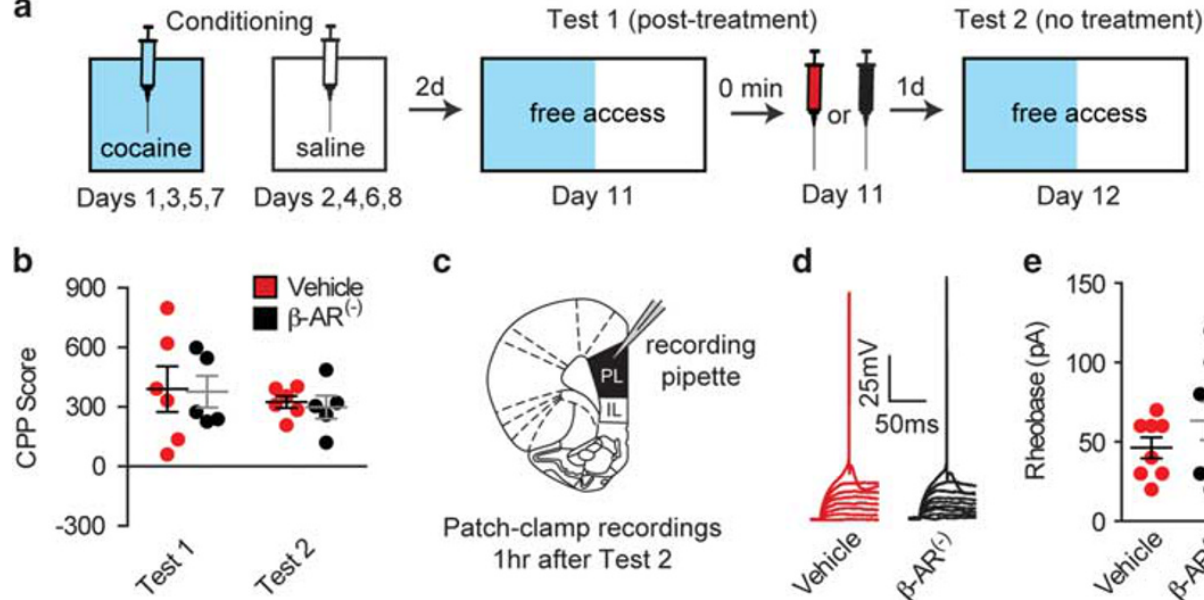

C

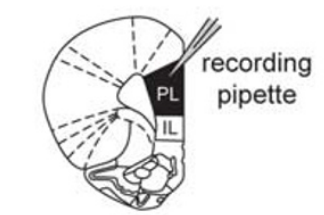

Patch-clamp recordings $1 \mathrm{hr}$ after Test 2 d

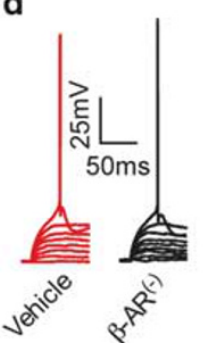

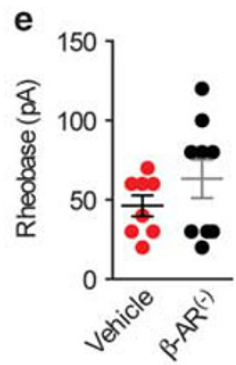

h

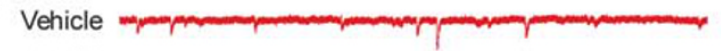

$\beta-A R^{(-)}$

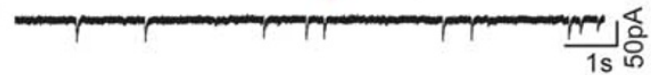

i

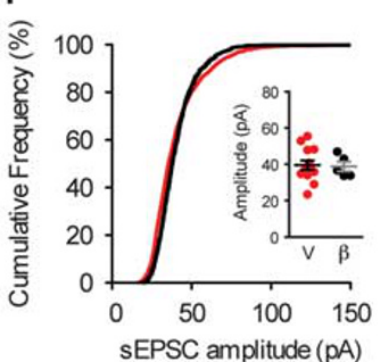

m

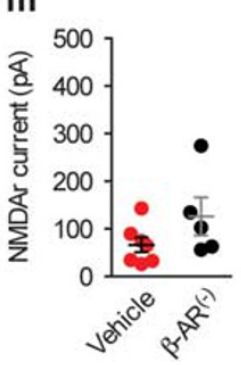

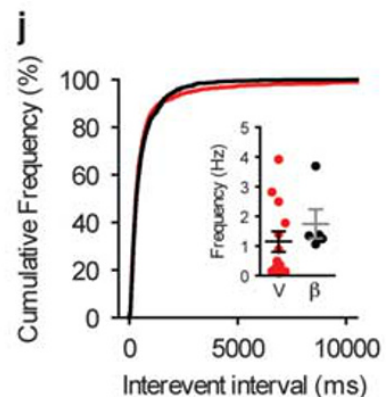

n

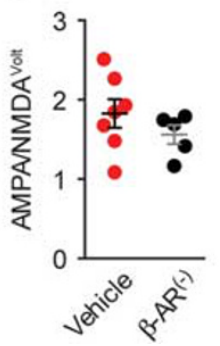

Figure $3 \quad \beta$-AR blockade after memory retrieval (during reconsolidation) has no effect on cocaine-evoked synaptic plasticity in PL-mPFC pyramidal neurons. (a) Schematic illustrating behavioral design of the experiment, wherein saline (red) or propranolol (black) was injected after the first CPP test. (b) Dot plots revealing no differences in CPP scores when propranolol was injected after the first CPP test (test I). (c) Coronal section illustrating patch-clamp recordings in PL-mPFC. (d) Representative waveforms for rheobase recordings. (e) Dot plots revealing that no difference in rheobase was found between groups. (f) Representative waveforms for excitability sweep recordings. (g) Line graph revealing that no differences in excitability sweeps were found between groups. ( $h$ ) Representative waveforms for sEPSC recordings. (i) Cumulative frequency distribution and dot plots (inset) revealing no differences in sEPSC amplitudes between groups. (j) Cumulative frequency distribution and dot plots (inset) revealing no differences in sEPSC frequencies between groups. ( $k$ ) Dot plots revealing that no difference in paired-pulse ratio was found between groups. (I) Dot plots revealing that no difference in AMPA receptor-mediated current was found between groups. $(m)$ Dot plots revealing that no difference in NMDA receptor-mediated current was found between groups. (n) Dot plots revealing that no difference in AMPA/NMDA ${ }^{\text {volt }}$ was found between groups. Lines in dot plots represent the mean $\pm \mathrm{SE}$.

group on NMDA receptor-mediated EPSCs $\left(\mathrm{F}_{2,25}=0.98\right.$, $p=0.39)$. These data suggest that AMPA receptor-mediated currents, but not NMDA receptor-mediated currents, are elevated following cocaine conditioning.

Although our preliminary recordings suggest that AMPA, but not NMDA receptor-mediated currents, are elevated following cocaine conditioning, these recordings can be problematic due to differences in the number of synapses that become activated during presynaptic stimulation. Thus, we confirmed the AMPA receptor plasticity by recording two different AMPA/NMDA ratios, one through voltage adjustment (AMPA/NMDA ${ }^{\text {Volt }}$ ) and the other through pharmacological isolation of the currents (AMPA/NMDA ${ }^{\text {Pharm; }}$; see 'Methods' section). Cocaine conditioning increased both 
AMPA/NMDA ${ }^{\text {Volt }}$ and AMPA/NMDA ${ }^{\text {Pharm, an effect that }}$ was reversed by propranolol treatment before the first CPP retrieval test (Figure $2 \mathrm{~g}$ and $\mathrm{h}$ ). For AMPA/NMDA ${ }^{\text {Volt }}$ (naive, $n=7$ cells, 3 rats; vehicle-treated, $n=9$ cells, 4 rats; propranolol-treated, $n=12$ cells, 4 rats), one-way ANOVA revealed a significant effect of group $\left(\mathrm{F}_{2,25}=14.80, p<0.001\right)$, and post hoc analyses revealed that neurons from vehicletreated rats had significantly higher AMPA/NMDA ${ }^{\text {Volt }}$ as compared with neurons from naive $(p<0.001)$ and propranolol-treated rats $(p<0.001)$. We next confirmed that AMPA/NMDA ${ }^{\text {Volt }}$ was accurately indexing the AMPA/ NMDA current ratio by pharmacologically isolating these currents. Indeed, for AMPA/NMDA ${ }^{\text {Pharm }}$ (naive, $n=4$ cells, 3 rats; vehicle-treated, $n=5$ cells, 4 rats; propranolol-treated, $n=3$ cells, 2 rats), one-way ANOVA revealed a significant effect of group $\left(\mathrm{F}_{2,9}=6.26, p=0.02\right)$, and post hoc analyses revealed that neurons from vehicle-treated rats had significantly higher AMPA/NMDA ${ }^{\text {Pharm }}$ as compared with neurons from naive $(p<0.01)$ and propranolol-treated rats $(p<0.05)$. Finally, in a subset of neurons, we performed both AMPA/NMDA ${ }^{\text {Volt }}$ and AMPA/NMDA ${ }^{\text {Pharm }}$ recordings, and found these ratios were positively correlated (Figure 2i; Pearson $r=0.93, p<0.001$ ), confirming accurate quantifications of AMPA/NMDA receptor currents. Collectively, these data reveal that AMPA receptor-mediated EPSCs in PL-mPFC pyramidal neurons are elevated following cocaine conditioning, and this postsynaptic plasticity is reversed by $\beta$-AR blockade during memory retrieval.

\section{$\beta$-AR Blockade during Reconsolidation Has No Effect on Cocaine-Associated Memory}

Here we show that cocaine-evoked synaptic plasticity in PL-mPFC can be reversed during retrieval, an effect that parallels long-lasting memory disruption. However, previous studies have shown that memories can also be impaired after retrieval, during memory reconsolidation (Clem and Huganir, 2010; Lee et al, 2005; Misanin et al, 1968; Nader et al, 2000; Nader and Hardt, 2009; Otis et al, 2015; Przybyslawski and Sara, 1997). Memory impairments related to retrieval and reconsolidation are mutually exclusive, as these phenomena involve distinct structures and behavioral characteristics (Otis et al, 2013, 2014a). Despite this, whether the effects of $\beta$-AR inhibition on cocaine-evoked synaptic plasticity are specific to retrieval, but not reconsolidation, is unknown. To assess this, rats were given systemic injections of the $\beta$-AR antagonist propranolol immediately after the first CPP retrieval test, during memory reconsolidation (Figure 3a; vehicle-treated, $n=6$ rats; propranolol-treated, $n=5$ rats). Consistent with previous reports (Fricks-Gleason and Marshall, 2008; Otis and Mueller, 2011), rats treated with a single systemic injection of propranolol after the first CPP retrieval test did not show a significantly reduced CPP during a subsequent propranolol-free test (Figure 3b). Two-way ANOVA revealed no effect of group $\left(\mathrm{F}_{1,9}=0.05, p=0.84\right)$, and no group by test interaction $\left(\mathrm{F}_{1,9}=0.01, p=0.92\right)$, confirming no effect of post-retrieval $\beta$-AR blockade on cocaine-associated memory reconsolidation.

\section{$\beta$-AR Blockade during Reconsolidation Has No Effect on Cocaine-Evoked Plasticity}

Next, we determined how $\beta$-AR blockade after retrieval affects cocaine-evoked plasticity in PL-mPFC. Rats that received post-retrieval vehicle or propranolol injections were killed after the second CPP test, and patch-clamp recordings were obtained from PL-mPFC pyramidal neurons (Figure 3c). Data revealed that post-retrieval propranolol did not affect the intrinsic excitability of PL-mPFC pyramidal neurons, as measured through rheobase (the minimum current required to evoke an action potential; Figure $3 \mathrm{~d}$ and e) and current-evoked excitability sweeps (Figure $3 \mathrm{f}$ and g). For rheobase recordings (vehicle-treated, $n=8$ cells, 4 rats; propranolol-treated, $n=9$ cells, 3 rats), an unpaired $t$-test revealed no effect $\left(t_{15}=1.19, p=0.25\right)$. For current-evoked excitability sweeps (vehicle-treated, $n=8$ cells, 4 rats; propranolol-treated, $n=9$ cells, 3 rats), twoway ANOVA revealed no effect of group $\left(F_{1,150}=0.88\right.$, $p=0.36)$, and no group by input interaction $\left(\mathrm{F}_{10,150}=0.23\right.$, $p=0.99$ ). Thus, post-retrieval $\beta$-AR blockade did not significantly influence the intrinsic excitability of PL-mPFC pyramidal neurons. Next, we evaluated the effects of postretrieval $\beta$-AR blockade on cocaine-evoked synaptic plasticity in PL-mPFC pyramidal neurons by recording sEPSCs (Figure $3 \mathrm{~h}$ ). Results show that post-retrieval $\beta$-AR blockade had no effect on sEPSC amplitude (Figure 3i) or sEPSC frequency (Figure 3j; vehicle-treated, $n=13$ cells, 5 rats; propranolol-treated, $n=5$ cells, 3 rats). For both measurements, $t$-tests revealed no effects between groups (sEPSC frequency: $t_{16}=0.93, p=0.36$; sEPSC amplitude: $t_{16}=0.16$, $p=0.87)$. Finally, we also determined the effects of postretrieval $\beta$-AR blockade on cocaine-evoked synaptic plasticity in PL-mPFC pyramidal neurons by measuring pairedpulse facilitation, AMPA receptor-mediated EPSCs, NMDA receptor-mediated EPSCs, and the AMPA/NMDA ratio. Data revealed that post-retrieval $\beta$-AR blockade had no effect on paired-pulse facilitation (Figure $3 \mathrm{k}$; vehicle-treated, $n=11$ cells, 5 rats; propranolol-treated, $n=7$ cells, 3 rats), and $t$-tests confirmed no effect between groups $\left(t_{16}=0.34\right.$, $p=0.74)$. In addition, there was no effect of post-retrieval $\beta$-AR blockade on AMPA receptor-mediated EPSCs, NMDA receptor-mediated EPSCs, or the AMPA/NMDA ratio (Figure 31, $\mathrm{m}$ and $\mathrm{n}$; vehicle-treated, $n=7$ cells, 4 rats; propranolol-treated, $n=5$ cells, 3 rats). For all measurements, $t$-tests revealed no effects between groups (AMPA: $t_{10}=1.55, p=0.15$; NMDA: $t_{16}=0.16, p=0.87$; AMPA/ NMDA $\left.{ }^{\text {Volt }}: t_{10}=1.12, p=0.29\right)$. Taken together, these data reveal that $\beta$-AR blockade after a single CPP memory retrieval test has no effect on memory and does not alter cocaine-evoked synaptic plasticity in PL-mPFC pyramidal neurons.

\section{DISCUSSION}

We characterized the effects of cocaine conditioning on presynaptic and postsynaptic efficacy in PL-mPFC. Conditioning increased sEPSC frequency and burst-firinginduced neural facilitation in PL-mPFC pyramidal neurons, suggesting stronger excitatory presynaptic drive onto these cells. Conditioning also increased sEPSC amplitude, AMPA receptor-mediated EPSCs, and AMPA:NMDA ratios, 
revealing greater excitatory postsynaptic efficacy in these neurons. The neuroadaptations were reversible in a retrievaldependent, timing-specific manner, as $\beta$-AR blockade before cocaine-associated memory retrieval, but not after, normalized presynaptic and postsynaptic neurotransmission in PL-mPFC. Considering that normalization of synaptic plasticity in PL-mPFC paralleled a persistent deficit in CPP memory, our data suggest that cocaine-evoked synaptic plasticity in PL-mPFC pyramidal neurons serves as a malleable retrieval mechanism for cocaine-associated memory.

\section{Drug-Evoked Plasticity in PL-mPFC Pyramidal Neurons}

Our findings add to a growing body of research showing that PL-mPFC is a critical module in addiction. In human addicts, the PFC is engaged by drug-associated cues, and the intensity of PFC activation correlates with reported drug cravings (Goldstein and Volkow, 2011; Grant et al, 1996; Grüsser et al, 2004; Kilts et al, 2001). In rodents, PL-mPFC neurons are engaged by cues that predict drug delivery (Ciccocioppo et al, 2001; Miller and Marshall, 2004,2005), whereas pharmacologic or optogenetic inhibition of PL-mPFC activity prevents cue-induced drug seeking (Hiranita et al, 2006; McLaughlin and See, 2003; Stefanik et al, 2013). Finally, previous studies show that PL-mPFC neurons undergo dendritic spine gain following drug exposure (Robinson et al, 2001; Robinson and Kolb, 2004,1999), an effect that correlates with the acquisition of drug-associated memory in vivo (Muñoz-Cuevas et al, 2013). This suggests that cocaine conditioning causes a persistent change in synaptic architecture in PL-mPFC, which allows persistent enhancement in functional synaptic connectivity (our findings). One important point to consider, however, is that in our study recordings were taken after CPP conditioning, and more specifically $1 \mathrm{~h}$ after a CPP retrieval test. Thus, it is possible that the synaptic plasticity observed is related to cocaine exposure, cocaine conditioning, or even cocaine-associated memory retrieval (although this is unlikely as cocaine evokes synaptic plasticity in the absence of retrieval; eg, see Muñoz-Cuevas et al, 2013; Robinson et al, 2001; Robinson and Kolb, 2004,1999). Taken together, these findings support the idea that synaptic plasticity in PL-mPFC is required for drug-associated memories, such that presentation of drug-associated cues can drive activity in PL-mPFC output neurons to engage drug seeking.

In contrast to synaptic plasticity, we did not observe any effects of cocaine conditioning on intrinsic excitability in PL-mPFC pyramidal neurons. This is at odds with some previous studies, which have found that repeated noncontingent cocaine delivery increases the excitability of these cells (Dong et al, 2005; Hearing et al, 2013; Nasif et al, 2005a, 2005b). In contrast to those experiments, rats that have undergone self-administration of cocaine in the presence of shock punishments have robustly diminished intrinsic excitability in PL-mPFC pyramidal neurons (Chen et al, 2013). Taken together, it is likely that intrinsic plasticity in PL-mPFC pyramidal neurons depends on the specific experimental protocol and/or the context in which cocaine was administered. For example, in studies wherein cocaine was experimenter delivered, the dose was higher $(15 \mathrm{mg} / \mathrm{kg}$, rather than $10 \mathrm{mg} / \mathrm{kg}$ presented here) and was given five times on consecutive days (Dong et al, 2005; Hearing et al, 2013; Nasif et al, 2005a, 2005b), rather than four times every other day (current data set). In addition, the method of delivery (ie, non-contingent $v s$ self-administration) is likely to influence how cocaine modifies the excitability of these cells. Taken together, the effects of cocaine exposure on intrinsic excitability in PL-mPFC pyramidal neurons has been mixed, and thus future research elucidating the context in which cocaine can modify the excitability of these cells is warranted.

Cocaine conditioning caused presynaptic plasticity in PL-mPFC, although the particular input(s) to PL-mPFC that is modified following conditioning is unclear. One likely candidate is the glutamatergic input from the basolateral amygdala (BLA). In support of this, the BLA becomes active upon presentation of drug-associated cues (Ciccocioppo et al, 2001), whereas optogenetic inactivation of BLA cell bodies or BLA terminals in PL-mPFC prevents cue-induced reinstatement of cocaine seeking (Stefanik and Kalivas, 2013). Furthermore, BLA neurons undergo dendritic spine plasticity in addiction, and pharmacological reversal of this plasticity causes long-lasting impairment in context-induced reinstatement of drug seeking (Young et al, 2016). Thus, plasticity at PL-mPFC presynaptic inputs from BLA may be a mechanism whereby cocaine-associated memories are stored. Next, presynaptic and postsynaptic plasticity in PL-mPFC neurons likely cause adaptations in the activity dynamics of distinct PL-mPFC output neurons. One likely PL-mPFC output is to the nucleus accumbens core (NAcc), as this pathway is activated by reward-predictive cues, and optogenetic inhibition of this activity reduces conditioned licking behavior (Otis et al, 2017). In addition, cocaine selfadministration leads to an enhancement in PL-mPFC excitatory synaptic drive onto NAcc neurons, and optogenetic inhibition of this plasticity prevents incubation of cocaine seeking (Ma et al, 2014). In addition, inactivation of PL-mPFC prevents drug-associated cues from driving glutamate release in the NAcc (LaLumiere and Kalivas, 2008), and inhibition of glutamate receptors in NAcc prevents cue-induced reinstatement of drug seeking (Di Ciano and Everitt, 2001; LaLumiere and Kalivas, 2008). Finally, optogenetic inhibition of PL-mPFC cell bodies, NAcc cell bodies, or PL-mPFC terminals in NAcc prevents cueand cocaine-induced reinstatement of cocaine seeking (Stefanik et al, 2013). Thus, our data showing cocaineevoked presynaptic and postsynaptic plasticity in PL-mPFC pyramidal neurons suggest that PL-mPFC input and outputs circuits, possibly involving the BLA and NAc, may be modified following drug experience. However, future studies are needed to identify how drug experience causes synaptic modifications within precise PFC circuits to control drug-associated memories.

\section{Maintenance of Cocaine-Associated Memory during Retrieval}

Cocaine conditioning induced plasticity in PL-mPFC circuits, and we found that this memory-related plasticity is reversible through $\beta$-AR receptor inhibition during memory retrieval, but not after retrieval (during reconsolidation). This data adds to a growing body of evidence showing that memory can be persistently impaired during retrieval, in 
the absence of any effects on reconsolidation. For example, in humans, inactivation of $\beta$-ARs before memory retrieval can lead to a sustained deficit in emotional memory expression (Kroes et al, 2010, 2016), effects that are associated with a sustained impairment in cue-induced activation of the PFC (Kroes et al, 2016). Similarly, in rodents, we have shown that systemic, intra-hippocampal, or intra-PFC injections of $\beta$-AR antagonists during retrieval, but not during reconsolidation, can persistently impair the expression of a cocaineinduced CPP, an effect that is not reversible with time or through a priming injection of cocaine (Fitzgerald et al, 2016; Otis and Mueller, 2011; Otis et al, 2013, 2014a). Furthermore, optogenetic inhibition of PFC output circuits can persistently impair cue-induced fear, whereas inhibition after retrieval has no effects (Do-Monte et al, 2015). Together, these data suggest that memory can be impaired during retrieval, prior to reconsolidation, and that the mechanisms that underlie memory deficits related to retrieval and reconsolidation are distinct.

It should be noted that it is possible that effects of systemic $\beta$-AR blockade on retrieval are due to $\beta$-AR antagonism in other regions, and not due to $\beta$-AR blockade in PL-mPFC. This is unlikely, however, for the following reasons. First, data reveal that systemic $\beta$-AR blockade prevents retrieval through central, but not peripheral, $\beta$-AR antagonism (Rodriguez-Romaguera et al, 2009; Otis and Mueller, 2011). Second, systemic $\beta$-AR antagonism reduces the activity of PL-mPFC neurons (Rodriguez-Romaguera et al, 2009), similar to what would be expected if $\beta$-AR blockers were acting directly in PL-mPFC (Mueller et al, 2008; Otis et al, 2013). Third, the behavioral effects of systemic $\beta$-AR blockade on cocaine-induced CPP memory retrieval can be fully replicated through $\beta$-AR blockade directly in PL-mPFC (Otis et al, 2013). Fourth, there are robust neural correlates of cocaine-induced CPP memory in PL-mPFC, both in vivo (Muñoz-Cuevas et al, 2013) and ex vivo (see Figures 1 and 2), and it is unclear why these neural correlates would be reversed by $\beta$-AR antagonism if the site of action was not, at least in part, in PL-mPFC. An alternative explanation for our effects is that the memory impairments observed here could be related to facilitated extinction learning, rather than longlasting impairments in memory retrieval. However, this caveat is also very unlikely as $\beta$-AR activation strengthens the formation of memory, including extinction learning (McGaugh, 2000; Mueller and Cahill, 2010). Furthermore, $\beta$-AR blockade impairs, rather than facilitates, extinction across multiple memory paradigms (Merlo and Izquierdo, 1967; LaLumiere and Kalivas, 2008; Mueller et al, 2008). Finally, memory retrieval impairments related to $\beta$-AR blockade prevent reinstatement of a cocaine-induced CPP (Otis and Mueller, 2011; Otis et al, 2014a), whereas extinction does not (Mueller and Stewart, 2000). Taken together, our data indicate that systemic $\beta$-AR blockade impairs cocaine-associated memory retrieval and cocaineevoked PL-mPFC synaptic plasticity through $\beta$-AR blockade in PL-mPFC.
Proposed Mechanism: $\beta$-AR Activation Maintains Memory during Retrieval by Preventing Spike-TimingDependent Depression at Modified PL-mPFC Synapses

Our data suggest that dynamic changes in PL-mPFC neurophysiology maintain cocaine-associated memory during retrieval, and the precise mechanisms that underlie this memory maintenance are beginning to be understood. We show that at the time of retrieval, PL-mPFC $\beta$-AR signaling is required for memory maintenance. $\beta$-AR activation rapidly elevates intrinsic excitability of PL-mPFC pyramidal neurons (Otis et al, 2013), and thus, PL-mPFC neuronal excitability is increased during retrieval. Intrinsic excitability provides signal amplification, indicating that excitatory synaptic inputs during retrieval would result in high frequency spiking of PL-mPFC pyramidal neurons. On the other hand, inhibition of PL-mPFC neuronal excitability would result in the absence of postsynaptic activity upon retrieval-related presynaptic input. Such neural asynchrony leads to spiketiming-dependent depression (Dan and Poo, 1992; Markram et al, 1997), a form of long-term depression that is controlled by noradrenergic signaling (Huang et al, 2013; Seol et al, 2007). Collectively, although this idea is speculative, our data suggest that PL-mPFC $\beta$-AR signaling increases intrinsic neuronal excitability during retrieval, and this may promote neuronal synchrony to prevent synaptic depression during retrieval. Considering this, future experimentation that identifies the specific mechanisms that allow $\beta$-AR blockade to persistently impair retrieval should be performed.

\section{FUNDING AND DISCLOSURE}

This study was supported by NIH R01-DA038042 (DM), NIH F32-DA041184 (JMO), and a grant from the Puerto Rico Science, Technology and Research Trust (DM). The authors declare no conflict of interest.

\section{ACKNOWLEDGMENTS}

We thank Dr Garret Stuber, Dr Gregory Quirk, and Dr Patrick Sheets for helpful suggestions on early versions of the manuscript. We also thank Jake Burkard, Matthew Drake, and Megha Sehgal for technical assistance.

\section{AUTHOR CONTRIBUTIONS}

JMO and DM designed experiments, interpreted the data, and wrote the manuscript. JMO performed the experiments and analyzed the data.

\section{REFERENCES}

Chen BT, Yau HJ, Hatch C, Kusumoto-Yoshida I, Cho SL, Hopf FW et al (2013). Rescuing cocaine-induced prefrontal cortex hypoactivity prevents compulsive cocaine seeking. Nature 496: 359-362.

Childress AR, McLellan AT, O’Brien CP (1986). Role of conditioning factors in the development of drug dependence. Psychiatr Clin North Am 9: 413-425.

Ciccocioppo R, Sanna PP, Weiss F (2001). Cocaine-predictive stimulus induces drug-seeking behavior and neural activation in limbic brain regions after multiple months of abstinence: reversal by D(1) antagonists. Proc Natl Acad Sci USA 98: 1976-1981. 
Clem RL, Huganir RL (2010). Calcium-permeable AMPA receptor dynamics mediate fear memory erasure. Science 330: 1108-1112.

Dan Y, Poo MM (1992). Hebbian depression of isolated neuromuscular synapses in vitro. Science 256: 1570-1573.

Di Ciano P, Everitt BJ (2001). Dissociable effects of antagonism of NMDA and AMPA/KA receptors in the nucleus accumbens core and shell on cocaine-seeking behavior. Neuropsychopharmacology 25: 341-360.

Do-Monte FH, Quiñones-Laracuente K, Quirk GJ (2015). A temporal shift in the circuits mediating retrieval of fear memory. Nature 519: 3607-3615.

Dong Y, Nasif FJ, Tsui JJ, Ju WY, Cooper DC, Hu XT et al (2005). Cocaine-induced plasticity of intrinsic membrane properties in prefrontal cortex pyramidal neurons: adaptations in potassium currents. J Neurosci 25: 936-940.

Fitzgerald MK, Otis JM, Mueller D (2016). Dissociation of $\beta 1$ - and $\beta 2$-adrenergic receptor subtypes in the retrieval of cocaineassociated memory. Behav Brain Res 296: 94-99.

Fricks-Gleason AN, Marshall JF (2008). Post-retrieval beta-adrenergic receptor blockade: effects on extinction and reconsolidation of cocaine-cue memories. Learn Mem 15: 643-648.

Goldstein RZ, Volkow ND (2011). Dysfunction of the prefrontal cortex in addiction: neuroimaging findings and clinical implications. Nat Rev Neurosci 12: 652-669.

Grant S, London ED, Newlin DB, Villemagne VL, Liu X, Contoreggi $C$ et al (1996). Activation of memory circuits during cue-elicited cocaine craving. Proc Natl Acad Sci USA 93: 12040-12045.

Grüsser SM, Wrase J, Klein S, Hermann D, Smolka MN, Ruf M et al (2004). Cue-induced activation of the striatum and medial prefrontal cortex is associated with subsequent relapse in abstinent alcoholics. Psychopharmacology 175: 296-302.

Hearing M, Kotecki L, Marron Fernandez de Velasco E, FajardoSerrano A, Chung HJ, Luján R et al (2013). Repeated cocaine weakens $\mathrm{GABA}(\mathrm{B})$-Girk signaling in layer $5 / 6$ pyramidal neurons in the prelimbic cortex. Neuron 80: 159-170.

Heather N, Stallard A, Tebbutt J (1991). Importance of substance cues in relapse among heroin users: comparison of two methods of investigation. Addict Behav 16: 41-49.

Hiranita T, Nawata Y, Sakimura K, Anggadiredja K, Yamamoto T (2006). Suppression of methamphetamine-seeking behavior by nicotinic agonists. Proc Natl Acad Sci USA 103: 8523-8527.

Huang S, Huganir RL, Kirkwood A (2013). Adrenergic gating of Hebbian spike-timing-dependent plasticity in cortical interneurons. J Neurosci 33: 13171-13178.

Kilts CD, Schweitzer JB, Quinn CK, Gross RE, Faber TL, Muhammad $\mathrm{F}$ et al (2001). Neural activity related to drug craving in cocaine addiction. Arch Gen Psychiatry 58: 334-341.

Kroes MCW, Strange BA, Dolan RJ (2010). Beta-adrenergic blockade during memory retrieval in humans evokes a sustained reduction of declarative emotional memory enhancement. J Neurosci 30: 3959-3963.

Kroes MCW, Tona KD, den Ouden HEM, Vogel S, van Wingen GA, Fernández G (2016). How administration of the beta-blocker propranolol before extinction can prevent the return of fear. Neuropsychopharmacology 41: 1569-1578.

LaLumiere RT, Kalivas PW (2008). Glutamate release in the nucleus accumbens core is necessary for heroin seeking. J Neurosci 28: 3170-3177.

Lee JLC, Di Ciano P, Thomas KL, Everitt BJ (2005). Disrupting reconsolidation of drug memories reduces cocaine-seeking behavior. Neuron 47: 795-801.

Ma YY, Lee BR, Wang X, Guo C, Liu L, Cui R et al (2014). Bidirectional modulation of incubation of cocaine craving by silent synapse-based remodeling of prefrontal cortex to accumbens projections. Neuron 83: 1453-1467.

Markram H, Lübke J, Frotscher M, Sakmann B (1997). Regulation of synaptic efficacy by coincidence of postsynaptic APs and EPSPs. Science 275: 213-215.
McGaugh JL (2000). Memory-a century of consolidation. Science 287: 248-251.

McLaughlin J, See RE (2003). Selective inactivation of the dorsomedial prefrontal cortex and the basolateral amygdala attenuates conditioned-cued reinstatement of extinguished cocaine-seeking behavior in rats. Psychopharmacology 168: 57-65.

Merlo AB, Izquierdo I (1967). The effect of catecholamines on learning in rats. Med Pharmacol Exp Int J Exp Med 16: 343-349.

Miller CA, Marshall JF (2005). Altered Fos expression in neural pathways underlying cue-elicited drug seeking in the rat. Eur $J$ Neurosci 21: 1385-1393.

Miller CA, Marshall JF (2004). Altered prelimbic cortex output during cue-elicited drug seeking. J Neurosci 24: 6889-6897.

Misanin JR, Miller RR, Lewis DJ (1968). Retrograde amnesia produced by electro-convulsive shock after reactivation of a consolidated memory trace. Science 160: 554-555.

Mueller D, Cahill SP (2010). Noradrenergic modulation of extinction learning and exposure therapy. Behav Brain Res 208: 1-11.

Mueller D, Porter JT, Quirk GJ (2008). Noradrenergic signaling in infralimbic cortex increases cell excitability and strengthens memory for fear extinction. J Neurosci 28: 369-375.

Mueller D, Stewart J (2000). Cocaine-induced conditioned place preference: reinstatement by priming injections of cocaine after extinction. Behav Brain Res 115: 39-47.

Muñoz-Cuevas FJ, Athilingam J, Piscopo D, Wilbrecht L (2013). Cocaine-induced structural plasticity in frontal cortex correlates with conditioned place preference. Nat Neurosci 16: 1367-1369.

Nader K, Hardt O (2009). A single standard for memory: the case for reconsolidation. Nat Rev Neurosci 10: 224-234.

Nader K, Schafe GE, Le Doux JE (2000). Fear memories require protein synthesis in the amygdala for reconsolidation after retrieval. Nature 406: 722-726.

Nasif FJ, Hu XT, White FJ (2005a). Repeated cocaine administration increases voltage-sensitive calcium currents in response to membrane depolarization in medial prefrontal cortex pyramidal neurons. J Neurosci 25: 3674-3679.

Nasif FJ, Sidiropoulou K, Hu XT, White FJ (2005b). Repeated cocaine administration increases membrane excitability of pyramidal neurons in the rat medial prefrontal cortex. J Pharmacol Exp Ther 312: 1305-1313.

Otis JM, Dashew KB, Mueller D (2013). Neurobiological dissociation of retrieval and reconsolidation of cocaine-associated memory. J Neurosci 33: 1271-1281a.

Otis JM, Fitzgerald MK, Mueller D (2014a). Inhibition of hippocampal $\beta$-adrenergic receptors impairs retrieval but not reconsolidation of cocaine-associated memory and prevents subsequent reinstatement. Neuropsychopharmacology 39: 303-310.

Otis JM, Fitzgerald MK, Mueller D (2014b). Infralimbic BDNF/ TrkB enhancement of GluN2B currents facilitates extinction of a cocaine-conditioned place preference. J Neurosci 34: 6057-6064.

Otis JM, Mueller D (2011). Inhibition of $\beta$-adrenergic receptors induces a persistent deficit in retrieval of a cocaine-associated memory providing protection against reinstatement. Neuropsychopharmacology 36: 1912-1920.

Otis JM, Namboodiri VMK, Matan AM, Voets ES, Mohorn EP, Kosyk O et al (2017). Prefrontal cortex output circuits guide reward seeking through divergent cue encoding. Nature 543: 103-107.

Otis JM, Werner CT, Mueller D (2015). Noradrenergic regulation of fear and drug-associated memory reconsolidation. Neuropsychopharmacology 40: 793-803.

Przybyslawski J, Sara SJ (1997). Reconsolidation of memory after its reactivation. Behav Brain Res 84: 241-246.

Robinson TE, Gorny G, Mitton E, Kolb B (2001). Cocaine selfadministration alters the morphology of dendrites and dendritic spines in the nucleus accumbens and neocortex. Synapse 39: 257-266.

Robinson TE, Kolb B (2004). Structural plasticity associated with exposure to drugs of abuse. Neuropharmacology 47: 33-46. 
Robinson TE, Kolb B (1999). Alterations in the morphology of dendrites and dendritic spines in the nucleus accumbens and prefrontal cortex following repeated treatment with amphetamine or cocaine. Eur J Neurosci 11: 1598-1604.

Rodriguez-Romaguera J, Sotres-Bayon F, Mueller D, Quirk GJ (2009). Systemic propranolol acts centrally to reduce conditioned fear in rats without impairing extinction. Biol Psychiatry 65: 887-892.

Seol GH, Ziburkus J, Huang S, Song L, Kim IT, Takamiya K et al (2007). Neuromodulators control the polarity of spike-timing-dependent synaptic plasticity. Neuron 55: 919-929.
Stefanik MT, Kalivas PW (2013). Optogenetic dissection of basolateral amygdala projections during cue-induced reinstatement of cocaine seeking. Front Behav Neurosci 7: 213.

Stefanik MT, Moussawi K, Kupchik YM, Smith KC, Miller RL, Huff ML et al (2013). Optogenetic inhibition of cocaine seeking in rats. Addict Biol 18: 50-53.

Young EJ, Blouin AM, Briggs SB, Sillivan SE, Lin L, Cameron MD et al (2016). Nonmuscle myosin IIB as a therapeutic target for the prevention of relapse to methamphetamine use. Mol Psychiatry 21: 615-623.

Zucker RS, Regehr WG (2002). Short-term synaptic plasticity. Annu Rev Physiol 64: 355-405. 